\title{
Adaptive Data Transmission in Multimedia Networks
}

\author{
Manimegalai Parry and N.Gangatharan \\ Centre for Multimedia Communications, Faculty of Engineering \\ Multimedia University, 63100 Cyberjaya, Selangor D.E, Malaysia
}

\begin{abstract}
This study suggests a method where the packet size of each source is adjusted according to the network bandwidth. A controller is used to trace the data transmission rate at the router. An algorithm is developed and coded in Tool Command Language. Simulation is performed on NS-2 using 4 different test cases and the results show that the proposed algorithm avoids router queue overflow.
\end{abstract}

Key words: Bandwidth, Data loss, NS-2, Queue overflow, Router

\section{INTRODUCTION}

Data loss due to overflow is presently a great challenge to network engineers. Therefore much attention is given to all these factors that contribute to such unwanted scenarios in the network transmission. When the total transmission rate is higher than the network bandwidth, packets are lost either because they are dropped when a router queue overflows or when a client buffer overflows [1,2]. Continuous media applications such as video and audio are sensitive not only to the packet loss probability but also to the correlation of packet losses $[1,4-6]$. Since the data packet size is directly proportional to the transmission rate, packet size adjustment is recommended to control the traffic flow. Much advancement is made to tolerate the need in current multimedia systems. Self adjusted network transmission has been proposed to optimize the network utilization and also to avoid packet overflow at the client buffer [2, 7, 9, 10]. Webcasting is introduced recently where producers bring real sound and vision to the web. Such broadcasting expects high Quality of Service (QoS) requirements regardless of network model, protocols or traffic agents used in a particular network [17, 19].

Much attention is given to high bandwidth requirement and real time delivery constraint. QoS with congestion control mechanism and end to end service guarantee and others have been introduced [11-14]. To enable satisfactory haptic multimedia data transmission, asynchronous transfer mode (ATM) defines constant bit rate $(\mathrm{CBR})$ to handle traffic at fixed rate and variable bit rate (VBR) service to handle varying traffic [3]. In this study, adaptive transmission rate is proposed using CBR traffic agent. A network model is designed with CBR and user datagram protocol (UDP) traffic agents with a single router. When the total transmission rate is greater than the network bandwidth, after the router, a control mechanism is used to determine the new packet size for each source and the transmission is reinitiated with total transmission rate that is equivalent to the network bandwidth. The simulation results showed that the proposed mechanism avoids router queue overflow.

\section{Adaptive Transmission Rate}

Network Model: A network model with 2 sources called node 0 and node 1 and two optional sinks called node 3 and node 4 are connected through a router called node 2 . The links between sources and the router are set to be $2 \mathrm{Mbps}$ with $10 \mathrm{~ms}$ of delay. The link between the router and the sink $\mathrm{n} 3$ is set to be $2 \mathrm{Mbps}$ with $10 \mathrm{~ms}$ of delay and the other link to the sink $\mathrm{n} 4$ is set to be 3 Mbps with $10 \mathrm{~ms}$ of delay. The designed network model is shown in Fig. 1.

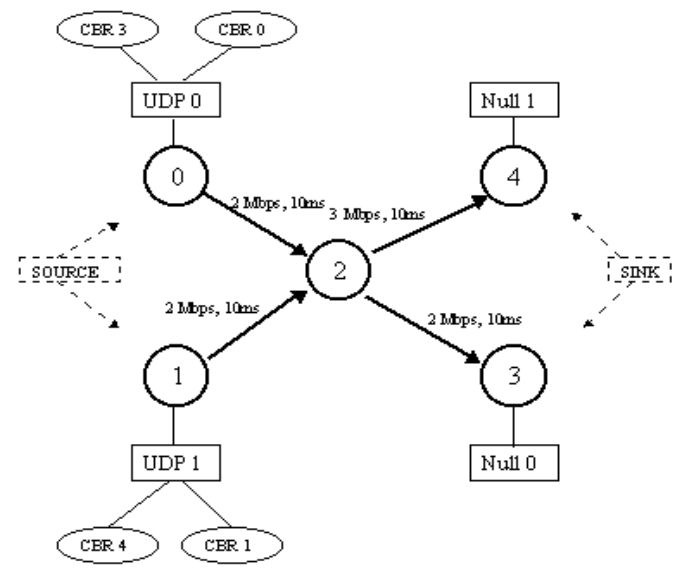

Fig. 1: Adaptive Transmission Rate Network Topology

Adaptive Transmission Rate Algorithm: A flowchart developed to describe the overall operation of the proposed network is illustrated in Fig. 2.

Concept: Standard sizes of packets are set at the both CBR0 and CBR1. When the transmission begins, both source nodes will begin transmission simultaneously. When the controller notices a possibility of overflow 


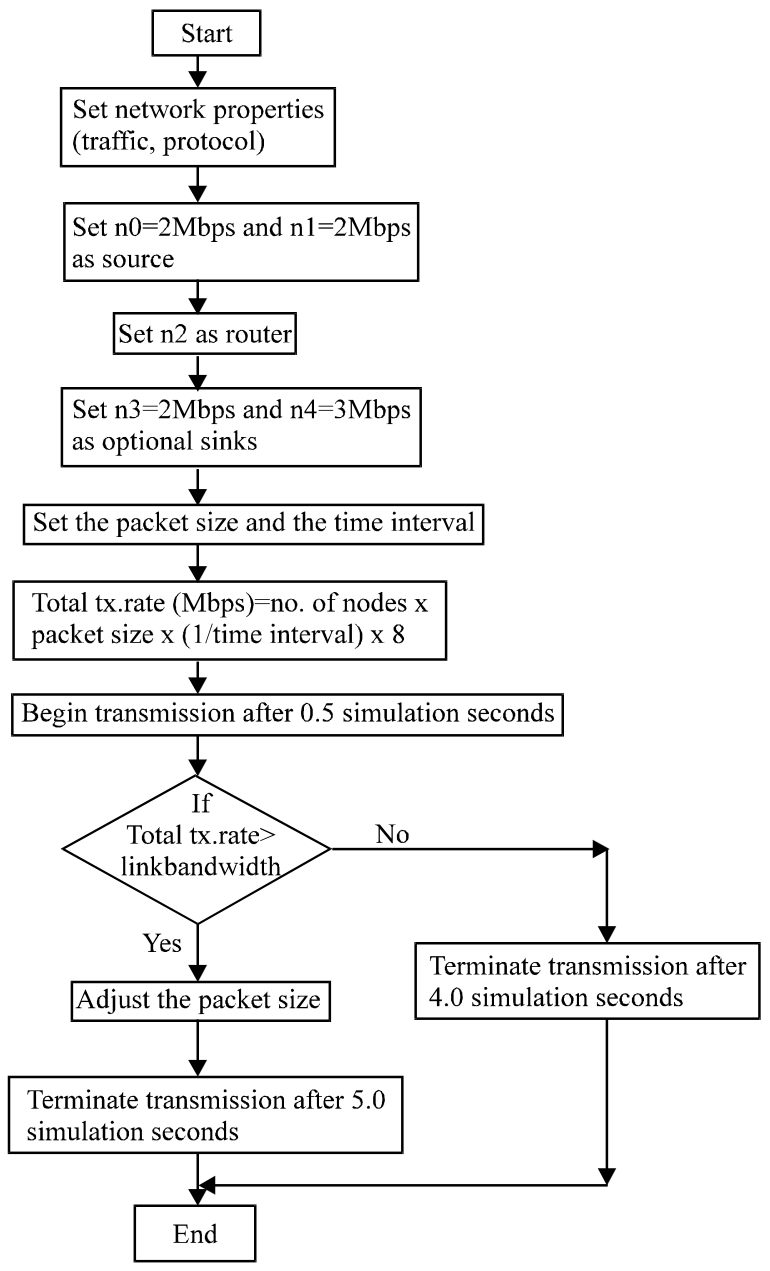

Fig. 2: Adaptive Transmission Rate Algorithm

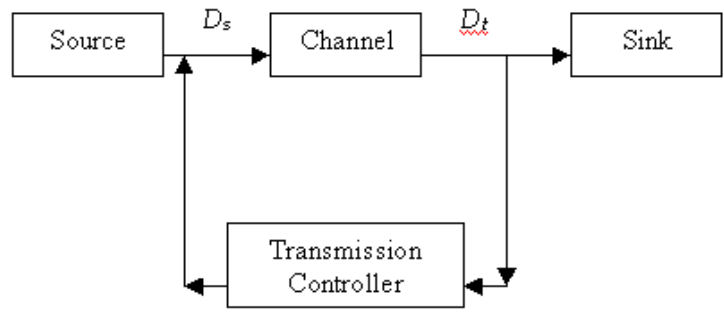

Fig. 3: Proposed Adaptive Transmission Rate Network Model

occurrence, it adjusts the packet size to be transmitted by the sources and alert the sources. The sources will reinitiate their transmission with adjusted sizes of packets using CBR3 and CBR4.

Pseudo code for rate adjustment for $n$ number source nodes on the network is given as follows. The controller only adjusts the transmission rate when there is a possibility of overflow. $D_{t}$ is the total transmission rate, $\mathrm{D}_{\mathrm{s}}$ is the transmission rate at the source and $\mathrm{BW}$ is the network bandwidth. The Fig. 3 shows the proposed network model.
$\mathrm{D}_{\mathrm{t}}=\mathrm{n} * \mathrm{Ds}$

If $\mathrm{D}_{\mathrm{t}}-\mathrm{BW}>0$

$\mathrm{C}=\left(\mathrm{D}_{\mathrm{t}}-\mathrm{BW}\right) /(\mathrm{n} * 1 /$ time_interval $* 8)$

New_packet_size $=$ Original_packet_size-C

CBR3 \& CBR4 = New_packet_size

Reinitialize transmission

Terminate tramission after 5

simulation seconds

End if

Pseudo Code that Describes the Packet Size Adjustment

\section{RESULTS}

Adaptive Transmission Data Rate Network: In this study, a simulation was performed to illustrate how the proposed adaptive transmission data rate mechanism is achieved in multimedia networks $[15,16]$. Simulation was carried out by first setting the node 3 as the sink and later by setting the node 4 as the sink. Simulation was run by setting the packet size that gives the accumulated data rate lower than the link bandwidth and the next by setting the packet size that gives the accumulated data rate higer than the link bandwidth.



Fig. 4: NS-2 Animator Screen Shot

Table 1 shows the test cases conducted on the same network. When $\mathrm{n} 3$ is set to be the sink, the network bandwidth is $2 \mathrm{Mbps}$ and when $\mathrm{n} 4$ is set to be the sink, the network bandwidth is 3 Mbps. The total transmission rate should not exceed the network bandwidth. If the total transmission rate is higher than the network bandwidth, the transmission controller adjusts the packet size of the source nodes so that the transmission rate is equal to the network bandwidth.

The plots show the total transmission rate vs. simulation time in the designed adaptive data transmission network. The plots for all 4 test cases are shown in Fig. 5-8.

Data Loss in a Non-Adaptive Transmission Data Rate Network: When the first two cases in Table 1 were 
Table 1: Test Cases for the experiment on Adaptive Transmission Network

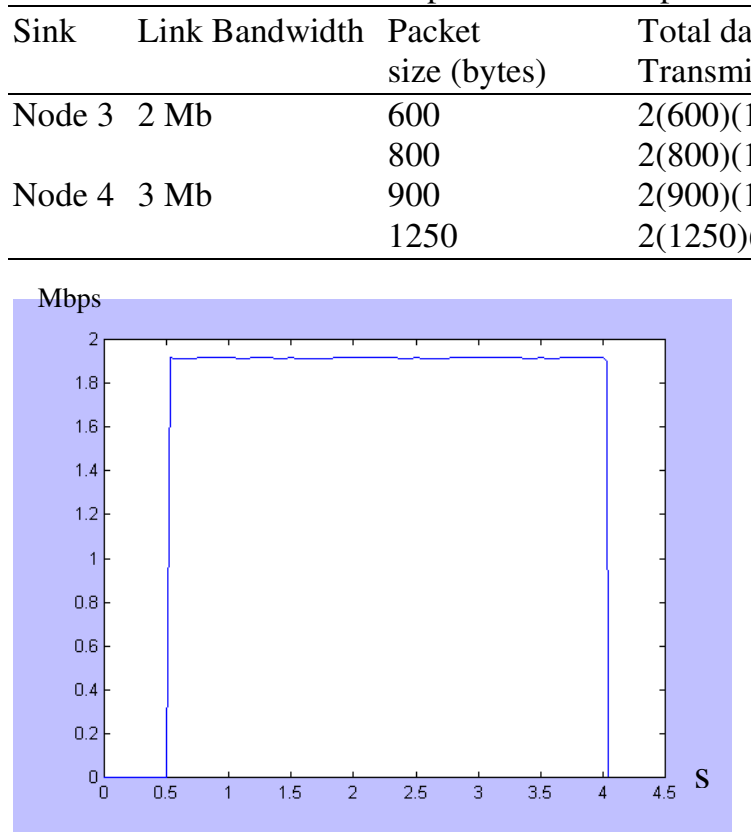

Fig. 5: Source Packet Size of 600 Bytes with Sink n3

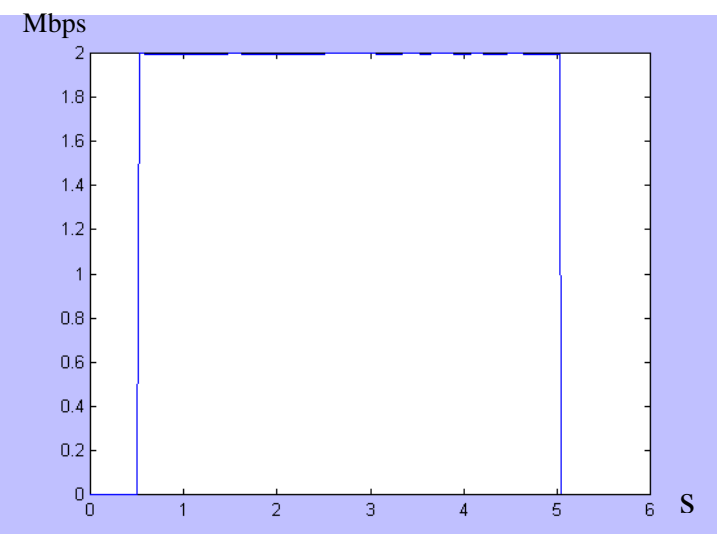

Fig. 6: Source Packet Size of 800 Bytes with Sink n3



Fig. 7: Source Packet Size of 900 Bytes with Sink n4

\begin{tabular}{ll}
$\begin{array}{l}\text { Adjusted data } \\
\text { transmission rate }\end{array}$ & $\begin{array}{l}\text { Stop } \\
\text { time (s) }\end{array}$ \\
\hline-- & 4.0 \\
$2 \mathrm{Mbps}$ & 5.0 \\
-- & 4.0 \\
$3 \mathrm{Mbps}$ & 5.0 \\
\hline
\end{tabular}

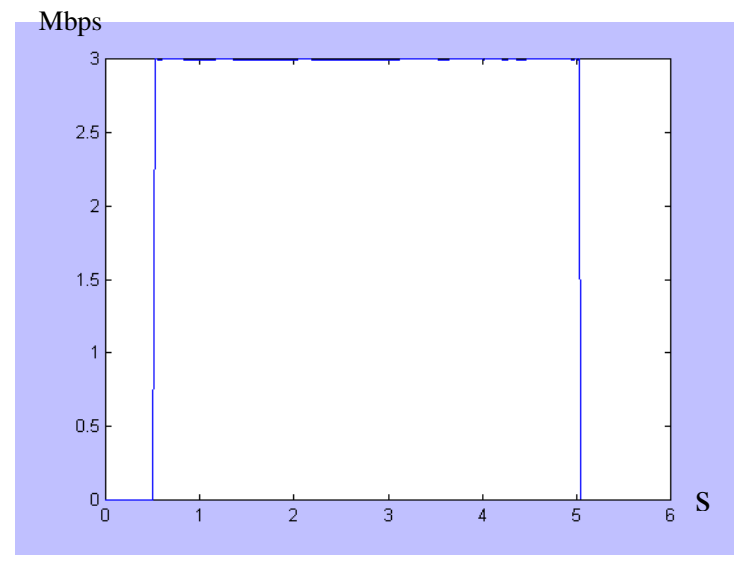

Fig. 8: Source Packet Size of 1250 Bytes with Sink n4

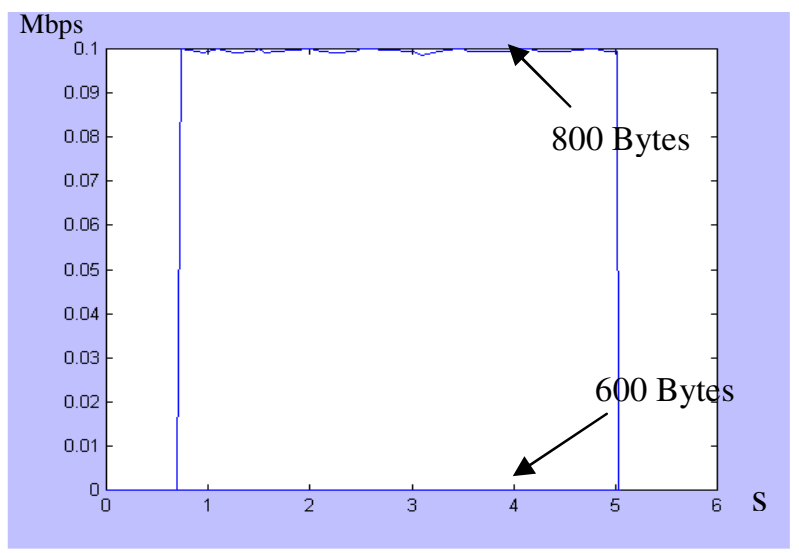

Fig. 9: Data Loss Rate with Sink n3

investigated without the control mechanism, some amount of data losses are traced when the total transmission rate exceed the network bandwidth. For the first case, when the sink node is set as $\mathrm{n} 3$ and the source packet size is 600 bytes, the total transmission rate is $1.92 \mathrm{Mbps}$. Since the total data transmission rate is within $2 \mathrm{Mbps}$, there is no data loss.

However, for the same sink node, when the source packet size is 800 bytes, some amount of data losses are traced because the total data transmission rate is 2.56 Mbps. Even though the difference is about $0.56 \mathrm{Mbps}$, only $0.1 \mathrm{Mbps}$ amount of losses are traced. That is due to the queue management at the router. 


\section{CONCLUSION}

There is a very high possibility for a router queue overflow to occur when video and audio data are transmitted over a network with low QoS management $[8,18]$. Router queue overflow occurs when the total data transmission rate is higher than the network bandwidth. This study, proposes an adaptive mechanism, that can avoid router queue overflow. A controller is used to monitor and adjust the data flow from the router to the sink. With such mechanism QoS requirements can be achieved by avoiding the occurrence of data losses.

\section{REFERENCES}

1. Henning, 2001. IP Networks: Compressed Video over Networks, Marcel Dekker. Inc. pp: 81-130.

2. Shyu, Chen and Luo, 2001. Self adjusted network transmission for multimedia data. Proceedings of the International Conference on Information Technology: Coding and Computing (ITCC'02).

3. Douglas, E.C., 2001. Computer Networks and Internet. $3^{\text {rd }}$ Edn. Prentice Hall.

4. Chen, S.C., M.L. Shyu and R.L. Kashyap, 2000. Augmented transition network as a semantic model for video data. Int. Networking and Inform. Sys., 3: 9- 25.

5. Chen, S.C., M.L. Shyu, C. Zhang and R.L. Kashyap, 2000. Object tracking and augmented transition network for video indexing and modeling. The $12^{\text {th }}$ IEEE Int. Conf. Tools with Artificial Intelligence (ICTAI 2000), Vancouver, British Columbia, Canada, pp: 428-435.

6. Chen, S.C., S. Sista, M.L. Shyu and R.L. Kashyap, 1999. Augmented transition networks as video browsing models for multimedia databases and multimedia information systems. $11^{\text {th }}$ IEEE Int. Conf. Tools with Artificial Intelligence (ICTAI'99), Chicago, IL, USA, pp: 175-182.

7. Effelsberg, W., O. Spaniol, A. Danthine and D. Ferrari, 1996. High-speed Networking For Multimedia Applications. Kluwer Academic Publishers.
8. Hui, J.Y., 1988. Resource allocation for broadband networks. IEEE J Selected Areas in Comm., pp: 1598-1608, 6.

9. Jain, R., 1996. Congestion control and traffic management in ATM networks: Recent advances and a survey. Computer Networks and ISDN Sys., 28: 1723-1738.

10. Kanakia, H., P. Mishra and A. Reibman, 1993. Adaptive congestion control scheme for real-time packet video transport. SIGCOMM Sym. on Comm. Architecture and Protocols. San Francisco, California, pp: 20-31, ACM/IEEE.

11. Lewis, F. and L. Syrmos, 1995. Optimal Control. John Wiley and Sons, Inc.

12. Garrett, M. and W. Willinger, 1994. Analysis, Modeling and Generation of Self-similar VBR Video Traffic. ACM SIGCOMM, pp: 269-280.

13. Ramanathan, S. and P.V. Rangan, 1993. Adaptive feedback techniques for synchronized multimedia retrieval over integrated networks. IEEE/ACM Trans. on Networking, 1: 246-260.

14. Roberts, J., U. Mocci and J. Virtamo (Eds.), 1996. Broadband Network Teletraffic. Final Report of Action Cost pp: 242, Springer.

15. Shyu, M.L., S.C. Chen and H. Luo, 2001. Optimal resource utilization in multimedia transmission. IEEE Int. Conf. on Multimedia and Expo (ICME), Waseda University, Tokyo, Japan, August 22- 25, pp: 880-883.

16. Steinmetz, R., 1996, Human perception of jitter and media synchronization. IEEE Selected Areas in Comm., 14: 61-72.

17. Tatipamula, M. and B. Khasnabish, 1998. Multimedia Communications Networks Technologies and Services. Artech House, pp: 133-173.

18. Wang, X. and H. Schulzrinne, 1999. Comparison of adaptive internet multimedia applications, IEICE Trans. Commun., E82-B: 806-818.

19. Woo, M., N.U. Qazi and A. Ghafoor, 1994. A synchronization framework for communication of pre-orchestrated multimedia information. IEEE Network, 8: 52-61. 